\title{
Estrategias de aprendizaje según los enfoques de aprendizaje en estudiantes del internado rotatorio de la Facultad de Medicina de la Universidad San Francisco Xavier de Chuquisaca (Sucre, Bolivia)
}

\author{
Ivonne F. Ramírez-Martínez, Germán Gallardo-Matienzo, Ángela Mita-Arancibia, Jesús F. Escanero-Marcén
} Objetivo. Analizar el uso de estrategias de aprendizaje según los enfoques de aprendizaje en estudiantes del internado
rotatorio de la Facultad de Medicina de la Universidad San Francisco Xavier de Chuquisaca (Sucre, Bolivia).

Sujetos y métodos. El estudio se realizó en 110 estudiantes con los cuestionarios ASSIST de Entwistle (1988), que valora los enfoques o aproximaciones hacia el estudio, y ACRA de Román y Gallego (1994), que analiza la preferencia en el uso de estrategias de adquisición, codificación, recuperación y apoyo.

Resultados. Los componentes de búsqueda de logros y monitorización de la efectividad definen la preferencia por el enfoque estratégico; en el profundo es la preferencia por el uso de la evidencias, pero en la aproximación superficial es el miedo al fracaso. Los enfoques estratégico y profundo presentan correlaciones significativas con las estrategias de adquisición como el repaso mental, repaso reiterado y subrayado lineal, y en la codificación, con las autopreguntas, aplicaciones y relaciones intracontenido. En estos enfoques hay correlación con todas las estrategias de recuperación, y en las de apoyo, con autoinstrucciones, automanejo-planificación y automanejo-regulación. El enfoque superficial no correlaciona con ninguna estrategia de codificación.

Conclusiones. A la vista de los resultados se abre un inusitado trabajo que comenzaría con el conocimiento de las aproximaciones del aprendiz y la dotación a los enfoques superficiales de un conjunto de estrategias que caracterizan a los profundos y estratégicos, con objeto de mejorar su aprendizaje.

Palabras clave. ACRA. Aprendizaje. ASSIST. Enfoques de aprendizaje. Estrategias de aprendizaje. Estudiantes de medicina.

Learning strategies according to learning approaches in students of the rotating internship of the Medicine School of the San Francisco Xavier of Chuquisaca University (Sucre, Bolivia)

Aim. To analyse the use of learning strategies according to the learning approaches of the medical students' (rotating internship) from University of San Francisco Xavier of Chuquisaca (Sucre, Bolivia).

Subjects and methods. The research was carried out with 110 rotating interns with the questionnaire ASSIST of Entwistle (1988), which assesses approaches to studying, and ACRA of Román and Gallego (1994) that analyses the preferences in the use of acquisition strategies, coding, recovery and support.

Results. The components of search achievements and monitoring effectiveness define the preference for the strategic approach. In the deep is the use of evidences but in the surface approximation is the fear of failure. The deep and strategic approaches present significant correlations with the acquisition strategies such as mental review, repeated review and linear underlined and in the coding with the self-questions, applications and inter content relations. In this approaches there is correlation with all recovery strategies and in the ones with autoinstructions, self-management/planning and selfmanagement/regulation. The superficial approach does not correlate with any coding strategy.

Conclusions. In view of the results, a conscious work is required with the aim of improving their learning. It could start with the knowledge of the learner approaches and the endowment of the superficial strategies with a set of strategies that distinguish the deep and strategic.

Key words. ACRA. ASSIST. Learning. Learning approaches. Learning strategies. Medical students.
Facultad de Ciencias y Tecnologías de la Salud (I.F. Ramírez-Martínez); Facultad de Psicología (G. GallardoMatienzo, A. Mita-Arancibia); Universidad San Francisco Xavier; Sucre, Bolivia. Facultad de Medicina; Universidad de Zaragoza (J.F. Escanero-Marcén); Zaragoza, España.

Correspondencia:

Dra. Ivonne Fabiana Ramírez Martínez. Facultad de Ciencias y Tecnologías de la Salud. Universidad San Francisco Xavier de Chuquisaca. San Alberto, 204. Casilla Postal 981. Sucre, Bolivia.

E-mail:

ifrm14@hotmail.com

Conflicto de intereses: No declarado.

Competing interests: None declared.

(c) 2015 FEM 


\section{Introducción}

La creación del Espacio Europeo de Educación Superior (EEES) conlleva el cambio del paradigma de la enseñanza superior que pasa de estar centrado en el profesor a estarlo en el alumno. Ello implica que el alumno asuma uno de los principios básicos de la formación continuada: aprender durante toda la vida (long-life learners). La transferencia de su propia construcción implica dotarlo de las herramientas más idóneas para ello y, especialmente, del conocimiento de sus propios procesos para poder elegir siempre el más óptimo y, en caso de que así no se haga, poder rectificar y cambiarlo [1].

De alguna manera, el plan de Bolonia ha mostrado claramente las dos caras de la moneda de la enseñanza universitaria: que la adquisición de las competencias nucleares o básicas de una carrera determinada se consiga de la mejor manera posible (metodologías activas, colaborativas, en pequeños grupos) y que el alumno disponga de todo lo concerniente con el aprendizaje (arsenal) para que durante toda la vida sea capaz de aprender significativamente, en cualquier contexto.

En el contexto universitario nacional de Bolivia, esta doble tarea no parece ni siquiera estar planteada: no es punto prioritario de la agenda científica el estudio de cómo facilitar el aprendizaje ni el haber superado metodologías de aprendizaje pasivo, centradas en el docente. El proceso de enseñanza se ejercita desde un rol tradicional de exposiciones magistrales que cuestionan la cultura de 'sentarse en aulas, escuchar pasivamente la lección, tomar apuntes, absorber, repetir y someterse a las pruebas periódicas para certificar su aprendizaje' [2]. La Universidad San Francisco Xavier de Chuquisaca, en la presente gestión, ha puesto en vigencia un nuevo modelo académico, donde señala que 'la formación profesional busca la práctica, la especialidad y la competencia para participar en un mundo cambiante; busca un individuo equilibrado en la teoría y la práctica' [3]. En este documento se señala además que en la producción del conocimiento intervienen el entendimiento, la observación, la imaginación, la voluntad, la memoria, la reflexión y la afectividad, donde el conocimiento también es colectivo, intersubjetivo y social. Sin embargo, las conclusiones de los diversos eventos de educación superior [4-6] señalan la necesidad de seguir optimizando los procesos para elevar la competencia del estudiante, de manera que permitan responder con mayor pertinencia a la misión y visión universitarias. Los estudios sobre el tema indican que se debe desarrollar una educación orientada en y para la creatividad como eje articulador para promover la autodeterminación del aprendizaje y la generación de innovación educativa [7].

Por otra parte, abordando la otra cara de Bolonia, desde diversas teorías de la psicología del aprendizaje se ha buscado explicar cómo el sujeto aprende un contenido. En ese sentido es importante que el profesorado comprenda que el aprendizaje es algo más, derivado de las actividades en las que el estudiante se implica, que el resultado de lo que el alumno es o de lo que el profesor hace [8]. Desde las teorías de la psicología constructivista, cuando se habla de aprendizaje uno de los conceptos más citados es el 'aprender a aprender' [9]; desde esta perspectiva se habla de los procesos cognitivos que señalan que el aprendizaje y la memoria se incorporan durante toda la vida, lo cual permite prepararse para una situación de respuesta ante un estímulo anteriormente vivido. Además, las teorías sociales constructivistas, como denominan algunos autores [10], han teorizado sobre la importancia de la autorregulación no sólo cognitiva, sino también emocional, conductual y contextual, lo que explica que el aprendizaje sea visto como un proceso complejo que seguirá desafiando a los investigadores actuales a buscar en los diferentes contextos, como comprender cada vez más los diversos mecanismos implicados en éste.

Desde este punto de vista, el papel que tienen los procesos de transformación y organización de la información, que ocurren en la mente de quien aprende, ha cobrado mucha importancia y, en consecuencia, la atención de los investigadores se ha dirigido a analizar las actividades que se realizan para aprender, retener y evocar [10], procesos fundamentales para el procesamiento cognitivo, donde están involucradas las estrategias de exploración, codificación y recuperación de la información. El aprendizaje, en suma, es un proceso activo que consiste en construir estructuras mentales o modificar o transformar las ya existentes a partir de las actividades mentales que se realizan, basadas en la activación y el uso del conocimiento previo [10]; sin embargo, la construcción y transformación de estas estructuras sólo es posible a través de estrategias que el sistema cognitivo emplea con el fin de optimizar al máximo el procesamiento de determinada información durante los procesos formales de aprendizaje, como es el caso de la formación universitaria. Y se destaca la importancia de las estrategias de aprendizaje con el objetivo de ir comprendiendo cada vez más cómo facilitar los procesos constructivistas de éste.

Se debe destacar hoy en día que el aprendizaje estratégico [9] se asume como uno de los principios psicopedagógicos que inspira el nuevo escenario de 
educación superior, pues tan importante como aprender unos contenidos determinados, lo es aprender y mejorar los procedimientos y estrategias que permiten continuar aprendiendo a lo largo de toda la vida y para que éste sea un proceso efectivo se requiere la aplicación flexible de un arsenal de estrategias [11].

Las estrategias de aprendizaje han sido estudiadas en el intento de comprender cada vez más el complejo proceso del aprendizaje y equivalen a la organización de los procesos mentales internos que, en calidad de variables intermedias, explican el curso y organización del pensamiento desde la recepción del dato o estímulo inicial hasta la respuesta final [12]. Monereo [13] señala que las estrategias de aprendizaje son procesos de toma de decisiones que se realizan de forma consciente y definida, a fin de alcanzar un objetivo, en los que se activan técnicas y procedimientos de distinta naturaleza (disciplinarios e interdisciplinarios) en un ambiente concreto y en una situación específica.

Las estrategias de aprendizaje [14] se aplican cuando se está asimilando la información contenida en un texto, en un artículo, en unos apuntes, es decir, en cualquier situación de estudio. El profesor debe conocer e intentar perfeccionar las estrategias de los buenos estudiantes (aprendedores profundos) e idear estrategias para que los alumnos peores (superficiales) las incorporen, perfeccionadas si es posible, a su trabajo.

Siguiendo esa línea teórica, el objetivo de este estudio es analizar el uso de estrategias de aprendizaje según los enfoques de aprendizaje de estudiantes del internado rotatorio de la Facultad de Medicina de la Universidad San Francisco Xavier de Chuquisaca y tratar de ver algunas regularidades en las relaciones entre ambos, al objeto de disponer de un material que ha de ayudar al profesorado a cumplir mejor con su papel.

\section{Sujetos y métodos}

\section{Descripción de la muestra}

En el estudio participaron 110 estudiantes, de ambos sexos (59 mujeres y 51 hombres), del internado rotatorio de la Facultad de Medicina de la Universidad San Francisco Xavier de Chuquisaca (Sucre, Bolivia). En la muestra se seleccionaron los estudiantes que habían aprobado todas las asignaturas correspondientes al quinto año y se excluyeron a aquellos con reprobaciones, segunda instancia o arrastre de materias.
El internado rotatorio es la última fase de la formación académica para la graduación del médico general. Se pretende que, de esta forma, los estudiantes desarrollen las suficientes habilidades y valores propios de la práctica laboral para incorporarlas al nivel de conocimiento científico que poseen, integrando los aspectos fundamentales del proceso educativo. Tiene una duración de un año y la formación práctica se realiza en los diferentes hospitales públicos de la ciudad, rotando bimestralmente por las especialidades del área de la medicina clínica y la medicina social.

Para la recogida de datos se avisó por parte de la Jefatura de Internado Rotatorio de la Facultad de Medicina al conjunto de estudiantes. Los instrumentos se aplicaron en una sola sesión a los internos del Hospital Santa Bárbara, Hospital Jaime Mendoza y Hospital Ginecoobstétrico de la ciudad de Sucre. Se administró el instrumento de enfoques de aprendizaje y, posteriormente, el cuestionario de valoración de estrategias. La duración media de la actividad fue de 45 minutos.

\section{Instrumentos utilizados}

\section{Determinación del enfoque de aprendizaje}

Para la determinación del enfoque de aprendizaje se aplicó el cuestionario ASSIST (Approaches and Study Skills Inventory for Students) de Entwistle [15]. El cuestionario evalúa el uso de estrategias de aprendizaje de los estudiantes en sus actividades de trabajo y la calidad del aprendizaje conseguido; contiene 52 ítems correspondientes a las tres categorías de enfoque analizadas: profundo, superficial y estratégico. Las características de cada enfoque son las siguientes:

- Enfoque profundo: búsqueda de significado, relación de ideas, uso de la evidencia, interés en las ideas.

- Enfoque estratégico: organización del estudio, gestión del tiempo, logros, monitorización de la efectividad.

- Enfoque superficial: falta de propósito, memorización sin relacionar, ley del mínimo esfuerzo, miedo al fracaso.

\section{Determinación de las estrategias de aprendizaje}

Para la determinación de las estrategias de aprendizaje se utilizó el cuestionario de adquisición, codificación, recuperación y apoyo (ACRA) de Román y Gallego [16] El cuestionario se diseñó para evaluar el grado en que el alumno posee y aplica las estrategias de aprendizaje; contiene un total de 32 ítems organizados en cuatro subescalas: 
Tabla I. Componentes por factores en los enfoques profundo, estratégico y superficial.

\begin{tabular}{|c|c|c|c|c|c|}
\hline & & $\begin{array}{l}\text { Profundos } \\
(n=27)\end{array}$ & $\begin{array}{l}\text { Estratégicos } \\
\quad(n=76)\end{array}$ & $\begin{array}{l}\text { Superficiales } \\
\qquad(n=5)\end{array}$ & $\begin{array}{c}\text { Total } \\
(n=108)\end{array}$ \\
\hline \multirow{4}{*}{$\begin{array}{l}\text { Enfoque } \\
\text { profundo }\end{array}$} & Búsqueda de significado & $15,52 \pm 0,56$ & & & $14,87 \pm 0,23$ \\
\hline & Relación de ideas & $15,30 \pm 0,50$ & & & $14,59 \pm 0,22$ \\
\hline & Uso de la evidencia & $16,22 \pm 0,38$ & & & $15,65 \pm 0,17$ \\
\hline & Interés en las ideas & $15,04 \pm 0,50$ & & & $14,55 \pm 0,21$ \\
\hline \multirow{5}{*}{$\begin{array}{l}\text { Enfoque } \\
\text { estratégico }\end{array}$} & Organización del estudio & & $15,45 \pm 0,26$ & & $14,93 \pm 0,23$ \\
\hline & Gestión del tiempo & & $15,37 \pm 0,28$ & & $14,81 \pm 0,25$ \\
\hline & $\begin{array}{l}\text { Atención a las } \\
\text { demandas de las tareas }\end{array}$ & & $15,86 \pm 0,28$ & & $15,28 \pm 0,26$ \\
\hline & Logros & & $16,70 \pm 0,22$ & & $16,16 \pm 0,22$ \\
\hline & $\begin{array}{l}\text { Monitorización } \\
\text { de la efectividad }\end{array}$ & & $16,43 \pm 0,24$ & & $16,19 \pm 0,20$ \\
\hline \multirow{4}{*}{$\begin{array}{l}\text { Enfoque } \\
\text { superficial }\end{array}$} & Falta de propósito & & & $14,00 \pm 0,84$ & $11,15 \pm 0,22$ \\
\hline & Memorizar sin relacionar & & & $14,80 \pm 0,58$ & $10,63 \pm 0,26$ \\
\hline & Ley del mínimo esfuerzo & & & $15,20 \pm 1,11$ & $11,50 \pm 0,26$ \\
\hline & Miedo al fracaso & & & $17,40 \pm 1,25$ & $14,06 \pm 0,27$ \\
\hline
\end{tabular}

- Adquisición: exploración, subrayado lineal, subrayado idiosincrático, epigrafiado, repaso en voz alta, repaso mental, repaso reiterado.

- Codificación: nemotécnicas, relaciones intracontenido, relaciones compartidas, imágenes, metáforas, aplicaciones, autopreguntas, paráfrasis, agrupamientos, secuencias, diagramas y mapas conceptuales.

- Recuperación: búsqueda de codificaciones, búsqueda de indicios, planificación de respuesta y respuesta escrita.

- Apoyo: autoconocimiento, automanejo, planificación, regulación, autoinstrucciones, autocontrol, contradistractoras, interacciones sociales y motivación intrínseca/extrínseca.

\section{Tratamiento estadístico}

Para procesar la información de los cuestionarios se elaboró una base de datos en el programa SPSS v. 18, para luego aplicar el coeficiente de correlación de Pearson $\left(r_{x y}\right)$-o coeficiente de correlación producto-momento de Pearson- como índice para la medida de la relación entre las dos variables de estudio: enfoques y estrategias de aprendizaje. Para la interpretación se tuvo en cuenta que los valores de la mencionada correlación oscilan entre -1 y 1 , donde 1 indica la existencia de una relación lineal positiva perfecta entre $x$ e $y, y-1$, la existencia de una relación lineal negativa perfecta entre $x$ e $y$. El valor 0 significa la no existencia de relación lineal entre $x$ e $y$. Se consideró significación estadística a partir de valores de $p \leq 0,05$.

\section{Resultados}

\section{Enfoques de aprendizaje}

Para analizar el uso de estrategias de aprendizaje en los estudiantes del internado rotatorio de la Facultad de Medicina de la Universidad San Francisco Xavier de Chuquisaca, en una primera etapa se procedió a identificar la preferencia que tienen por la aplicación de determinado enfoque de aprendizaje y se encontró que existe mayor prevalencia por el enfoque estratégico $(69,1 \%)$, seguido del enfoque profundo $(24,5 \%)$ y del enfoque superficial $(4,5 \%)$. Con respecto al sexo, de los 50 estudiantes varones se observó que 36 tenían mayor orientación por el enfoque estratégico, 12 por el profundo y dos por el superficial; en el caso del sexo femenino, los valores son similares: 40 estudiantes con mayor orientación por el enfoque estratégico, 15 por el profundo y tres por el superficial, por lo que $\chi_{(2)}^{2}=0,152(p \geq 0,927)$, lo que indica que en la muestra de estudio no existen diferencias por sexo en cuanto al tipo de enfoque que aplican los estudiantes.

Los componentes por factores en los enfoques estratégico, profundo y superficial se muestran en la tabla I, donde se expresan los resultados como media \pm desviación estándar. Finalmente, debe indicarse que un parámetro de comparación válido sería la media teórica. Dicho valor se ha calculado promediando el valor más bajo y el más alto posibles; dado que cada componente está compuesto por cuatro ítems y la escala va desde 1 (nunca) hasta 5 (siempre), se tiene:

$$
\text { Media teórica }=\frac{(1 \times 4)+(5 \times 4)}{2}=\frac{4+20}{2}=12 \text {, }
$$

lo que significa que aquellos valores superiores a 12 están por encima de la media teórica, indicando que ese componente se utiliza de manera frecuente.

En la tabla I, respecto al grupo de estudiantes que aplican con mayor preferencia un enfoque estraté- 
gico, se puede señalar que el componente 'logros', seguido de 'monitorización de la efectividad', puntúan con los valores más altos, lo que permite concluir que son las estrategias más utilizadas por estos estudiantes.

En cuanto a los estudiantes que aplican un enfoque profundo es el componente 'uso de la evidencia' el que tiene el promedio más alto y, en consecuencia, el más utilizado por estos estudiantes.

En el grupo de estudiantes que con mayor preferencia aplican un enfoque superficial es el componente 'miedo al fracaso' el que tiene el promedio más alto, debiéndose indicar en este caso que el error es considerable porque la muestra es pequeña (cinco personas), por lo que resultaría interesante su análisis en muestras mayores.

\section{Correlaciones entre enfoques \\ y estrategias de aprendizaje}

Para el análisis de correlaciones se han construido las tablas siguientes, donde se indican las correlaciones de Pearson entre los enfoques y las estrategias de aprendizaje. En el caso de los enfoques hay tres categorías (profundo, estratégico y superficial), mientras que las estrategias se encuentran agrupadas en estrategias de adquisición de información, codificación de información, recuperación de información y apoyo al procesamiento, por lo que la matriz obtenida es de $4 \times 3$.

En la tabla II se observa que las únicas dos variables que no presentan una correlación lineal son el aprendizaje superficial y la codificación de información $\left(r_{x y}=0,120\right)$.

A continuación se desglosan separadamente las estrategias de cada subescala del instrumento ACRA, donde se realiza un análisis detallado de las siete estrategias correspondientes a adquisición de información, 12 correspondientes a codificación de información, cuatro correspondientes a recuperación de información y nueve a apoyo al procesamiento.

\section{Correlaciones entre enfoques y}

\section{estrategias de adquisición de información}

En la tabla III se describen las correlaciones de los enfoques de aprendizaje con respecto a las estrategias utilizadas por los estudiantes para la adquisición de la información.

Se puede advertir que los enfoques de aprendizaje profundo y estratégico se correlacionan con todas las estrategias de adquisición de información, siendo las más elevadas las relaciones con el repaso mental, repaso reiterado y subrayado lineal. El enfoque superficial no tiene relación con las estrate-
Tabla II. Enfoques de aprendizaje y estrategias de aprendizaje.

\begin{tabular}{|c|c|c|c|c|}
\hline & & $\begin{array}{l}\text { Aprendizaje } \\
\text { profundo }\end{array}$ & $\begin{array}{l}\text { Aprendizaje } \\
\text { estratégico }\end{array}$ & $\begin{array}{c}\text { Aprendizaje } \\
\text { superficial }\end{array}$ \\
\hline \multirow{2}{*}{$\begin{array}{l}\text { Adquisición } \\
\text { de información }\end{array}$} & Correlación de Pearson & $0,520^{a}$ & $0,493^{a}$ & $0,270^{a}$ \\
\hline & Significación (bilateral) & 0,000 & 0,000 & 0,004 \\
\hline \multirow{2}{*}{$\begin{array}{l}\text { Codificación } \\
\text { de información }\end{array}$} & Correlación de Pearson & $0,605^{a}$ & $0,534^{a}$ & 0,120 \\
\hline & Significación (bilateral) & 0,000 & 0,000 & 0,212 \\
\hline \multirow{2}{*}{$\begin{array}{l}\text { Recuperación } \\
\text { de información }\end{array}$} & Correlación de Pearson & $0,557^{a}$ & $0,477^{a}$ & $0,274^{a}$ \\
\hline & Significación (bilateral) & 0,000 & 0,000 & 0,004 \\
\hline \multirow{2}{*}{$\begin{array}{l}\text { Apoyo al } \\
\text { procesamiento }\end{array}$} & Correlación de Pearson & $0,691^{a}$ & $0,606^{a}$ & $0,268^{a}$ \\
\hline & Significación (bilateral) & 0,000 & 0,000 & 0,005 \\
\hline
\end{tabular}

a Correlación significativa al nivel 0,01 (bilateral).

Tabla III. Enfoques y estrategias de adquisición de información.

\begin{tabular}{|c|c|c|c|c|}
\hline & & $\begin{array}{c}\text { Aprendizaje } \\
\text { profundo }\end{array}$ & $\begin{array}{l}\text { Aprendizaje } \\
\text { estratégico }\end{array}$ & $\begin{array}{c}\text { Aprendizaje } \\
\text { superficial }\end{array}$ \\
\hline \multirow{2}{*}{ Exploración } & Correlación de Pearson & $0,224^{b}$ & $0,189^{b}$ & $0,310^{a}$ \\
\hline & Significación (bilateral) & 0,019 & 0,048 & 0,001 \\
\hline \multirow{2}{*}{$\begin{array}{l}\text { Subrayado } \\
\text { lineal }\end{array}$} & Correlación de Pearson & $0,412^{a}$ & $0,398^{a}$ & $0,222^{b}$ \\
\hline & Significación (bilateral) & 0,000 & 0,000 & 0,020 \\
\hline \multirow{2}{*}{$\begin{array}{l}\text { Subrayado } \\
\text { idiosincrático }\end{array}$} & Correlación de Pearson & $0,288^{a}$ & $0,306^{a}$ & 0,082 \\
\hline & Significación (bilateral) & 0,002 & 0,001 & 0,392 \\
\hline \multirow{2}{*}{ Epigrafiado } & Correlación de Pearson & $0,341^{a}$ & $0,342^{a}$ & 0,139 \\
\hline & Significación (bilateral) & 0,000 & 0,000 & 0,146 \\
\hline \multirow{2}{*}{$\begin{array}{l}\text { Repaso } \\
\text { en voz alta }\end{array}$} & Correlación de Pearson & $0,296^{a}$ & $0,211^{a}$ & $0,362^{a}$ \\
\hline & Significación (bilateral) & 0,002 & 0,027 & 0,000 \\
\hline \multirow{2}{*}{$\begin{array}{l}\text { Repaso } \\
\text { mental }\end{array}$} & Correlación de Pearson & $0,536^{a}$ & $0,582^{a}$ & $-0,035$ \\
\hline & Significación (bilateral) & 0,000 & 0,000 & 0,717 \\
\hline \multirow{2}{*}{$\begin{array}{l}\text { Repaso } \\
\text { reiterado }\end{array}$} & Correlación de Pearson & $0,521^{a}$ & $0,452^{a}$ & $0,273^{a}$ \\
\hline & Significación (bilateral) & 0,000 & 0,000 & 0,004 \\
\hline
\end{tabular}

${ }^{a}$ Correlación significativa al nivel 0,01 (bilateral); ${ }^{b}$ Correlación significativa al nivel 0,05 (bilateral). 
Tabla IV. Enfoques de aprendizaje y estrategias de codificación de información.

\begin{tabular}{|c|c|c|c|c|}
\hline & & $\begin{array}{l}\text { Aprendizaje } \\
\text { profundo }\end{array}$ & $\begin{array}{l}\text { Aprendizaje } \\
\text { estratégico }\end{array}$ & $\begin{array}{l}\text { Aprendizaje } \\
\text { superficial }\end{array}$ \\
\hline \multirow{2}{*}{ Nemotecnias } & Correlación de Pearson & $0,329^{a}$ & $0,334^{a}$ & 0,119 \\
\hline & Significación (bilateral) & 0,000 & 0,000 & 0,215 \\
\hline \multirow{2}{*}{$\begin{array}{l}\text { Relaciones } \\
\text { intracontenido }\end{array}$} & Correlación de Pearson & $0,537^{a}$ & $0,482^{a}$ & 0,044 \\
\hline & Significación (bilateral) & 0,000 & 0,000 & 0,647 \\
\hline \multirow{2}{*}{$\begin{array}{l}\text { Relaciones } \\
\text { compartidas }\end{array}$} & Correlación de Pearson & $0,506^{a}$ & $0,475^{a}$ & $-0,027$ \\
\hline & Significación (bilateral) & 0,000 & 0,000 & 0,777 \\
\hline \multirow{2}{*}{ Imágenes } & Correlación de Pearson & $0,524^{a}$ & $0,370^{a}$ & 0,149 \\
\hline & Significación (bilateral) & 0,000 & 0,000 & 0,121 \\
\hline \multirow{2}{*}{ Metáforas } & Correlación de Pearson & $0,433^{a}$ & $0,291^{a}$ & 0,078 \\
\hline & Significación (bilateral) & 0,000 & 0,002 & 0,419 \\
\hline \multirow{2}{*}{ Aplicaciones } & Correlación de Pearson & $0,598^{a}$ & $0,541^{a}$ & $-0,003$ \\
\hline & Significación (bilateral) & 0,000 & 0,000 & 0,975 \\
\hline \multirow{2}{*}{ Autopreguntas } & Correlación de Pearson & $0,599^{a}$ & $0,500^{a}$ & 0,104 \\
\hline & Significación (bilateral) & 0,000 & 0,000 & 0,278 \\
\hline \multirow{2}{*}{ Paráfrasis } & Correlación de Pearson & $0,416^{a}$ & $0,362^{a}$ & 0,105 \\
\hline & Significación (bilateral) & 0,000 & 0,000 & 0,276 \\
\hline \multirow{2}{*}{ Agrupamientos } & Correlación de Pearson & $0,367^{a}$ & $0,383^{a}$ & 0,125 \\
\hline & Significación (bilateral) & 0,000 & 0,000 & 0,193 \\
\hline \multirow{2}{*}{ Secuencias } & Correlación de Pearson & $0,377^{\mathrm{a}}$ & $0,294^{a}$ & 0,137 \\
\hline & Significación (bilateral) & 0,000 & 0,002 & 0,153 \\
\hline \multirow{2}{*}{$\begin{array}{l}\text { Mapas } \\
\text { conceptuales }\end{array}$} & Correlación de Pearson & $0,355^{a}$ & $0,324^{a}$ & 0,133 \\
\hline & Significación (bilateral) & 0,000 & 0,001 & 0,166 \\
\hline \multirow{2}{*}{ Diagramas } & Correlación de Pearson & $0,367^{a}$ & $0,307^{a}$ & 0,106 \\
\hline & Significación (bilateral) & 0,000 & 0,001 & 0,271 \\
\hline
\end{tabular}

a Correlación significativa al nivel 0,01 (bilateral).

\section{Correlaciones entre enfoques y} estrategias de codificación de información

En la tabla IV se describen las correlaciones de los enfoques de aprendizaje con respecto a las estrategias utilizadas por los estudiantes para la codificación de la información.

Como se observa, los enfoques de aprendizaje profundo y estratégico se correlacionan con todas las estrategias de codificación de información, siendo las más intensas las relaciones con autopreguntas, aplicaciones y relaciones intracontenido. El enfoque superficial no tiene relación con ninguna estrategia de codificación de información.

\section{Correlaciones entre enfoques y estrategias de recuperación de información}

En la tabla V se describen las correlaciones de los enfoques de aprendizaje respecto a las estrategias utilizadas por los estudiantes para la recuperación de la información.

Se observa que los enfoques de aprendizaje profundo y estratégico se correlacionan con todas las estrategias de recuperación de información en similar magnitud, mientras que el enfoque superficial no tiene relación con la estrategia de planificación de respuesta y sí con las demás.

\section{Correlaciones entre enfoques y estrategias de apoyo al procesamiento de la información}

En la tabla VI se describen las correlaciones de los enfoques de aprendizaje respecto a las estrategias de apoyo al procesamiento.

Como puede observarse, el enfoque de aprendizaje profundo se correlaciona con todas las estrategias de apoyo al procesamiento, mientras que el enfoque de aprendizaje estratégico no presenta relación con la estrategia de autocontrol, y el enfoque de aprendizaje superficial, con las estrategias de automanejo/planificación, autoinstrucciones, contradistractoras y motivación extrínseca e intrínseca. Tanto el enfoque de aprendizaje profundo como el estratégico presentan como relaciones más intensas las referidas a autoinstrucciones, automanejo/planificación y automanejo/regulación y evaluación. Finalmente se observa que el enfoque de aprendizaje superficial presenta relaciones más intensas con estrategias como motivación de escape y autoconocimiento.

En suma, las correlaciones entre los enfoques estratégico y profundo y las estrategias de codificación son bastante significativas; por el contrario, en el enfoque superficial no se correlacionan. También es de destacar que los enfoques estratégico y profundo se correlacionan de forma intensa con las es- 
trategias de recuperación, mientras que los estudiantes con preferencia por la aproximación superficial lo hacen con menos intensidad al no aplicar estrategias metacognitivas, como es el caso de la planificación de la respuesta, que resulta ser una estrategia clave para una recuperación más efectiva de la información.

\section{Discusión}

Como se indicó en una publicación previa [17], una buena aproximación al análisis de los enfoques consiste en no etiquetar a cada estudiante con un enfoque de aprendizaje inmutable, sino que es el diseño de las oportunidades de aprendizaje lo que incentiva al estudiante a adoptar un enfoque particular. De acuerdo con Biggs et al [18], estos análisis deben entenderse como que esta interacción entre las características del estudiante y el contexto o ambiente de estudio es la que lo conduce a elegir diferentes enfoques.

Para Esquivel et al [19], la perspectiva teórica del enfoque al aprendizaje del estudiante postuló que el entendimiento del aprendizaje debería ser examinado a través de las experiencias del aprendiz. La forma en que el estudiante aprende se relaciona con las características y cantidad de tareas solicitadas en los cursos, con el contexto educativo y con sus motivos, que a su vez influyen en las estrategias de aprendizaje. Como señalan estos autores, si bien existen investigaciones para conocer los enfoques al aprendizaje en diferentes contextos educativos y campos disciplinares específicos, en Latinoamérica hay escasa investigación al respecto, así como en otras partes del mundo, y más en lo que a medicina se refiere.

Comprender cómo el estudiante universitario del internado rotatorio de medicina en nuestro medio se aproxima al aprendizaje es una temática abordada en estudios de otros países y para desarrollar esta investigación se ha considerado la revisión de investigaciones previas sobre el tema [7,17,20-22], donde se analizó la importancia del contexto como variable importante en el proceso del aprendizaje.

Inicialmente, en el grupo estudiado no se han encontrado diferencias por sexos, resultados que difieren con los encontrados por Salas [21], quien señaló que el sexo femenino se relaciona más con el enfoque profundo. En el grupo de la Universidad San Francisco Xavier, al parecer, resulta más determinante para su preferencia por el enfoque estratégico el nivel que cursan, hecho que ya se estudió en otros contextos [22], donde se comunicó la relación entre la orientación estratégica y los niveles de formación. Tratándose de que estos estudiantes cursan el último
Tabla V. Enfoques y estrategias de recuperación de información.

\begin{tabular}{|c|c|c|c|c|}
\hline & & $\begin{array}{l}\text { Aprendizaje } \\
\text { profundo }\end{array}$ & $\begin{array}{l}\text { Aprendizaje } \\
\text { estratégico }\end{array}$ & $\begin{array}{c}\text { Aprendizaje } \\
\text { superficial }\end{array}$ \\
\hline \multirow{2}{*}{$\begin{array}{l}\text { Búsqueda de } \\
\text { codificaciones }\end{array}$} & Correlación de Pearson & $0,434^{a}$ & $0,419^{a}$ & $0,264^{a}$ \\
\hline & Significación (bilateral) & 0,000 & 0,000 & 0,005 \\
\hline \multirow{2}{*}{$\begin{array}{l}\text { Búsqueda } \\
\text { de indicios }\end{array}$} & Correlación de Pearson & $0,445^{a}$ & $0,341^{a}$ & $0,245^{a}$ \\
\hline & Significación (bilateral) & 0,000 & 0,000 & 0,010 \\
\hline \multirow{2}{*}{$\begin{array}{l}\text { Planificación } \\
\text { de respuesta }\end{array}$} & Correlación de Pearson & $0,496^{a}$ & $0,404^{a}$ & 0,160 \\
\hline & Significación (bilateral) & 0,000 & 0,000 & 0,096 \\
\hline \multirow{2}{*}{$\begin{array}{l}\text { Respuesta } \\
\text { escrita }\end{array}$} & Correlación de Pearson & $0,521^{a}$ & $0,462^{a}$ & $0,260^{a}$ \\
\hline & Significación (bilateral) & 0,000 & 0,000 & 0,006 \\
\hline
\end{tabular}

a Correlación significativa al nivel 0,01 (bilateral).

año, su aprendizaje se relaciona con la práctica profesional, lo que al parecer determina mayor orientación estratégica independientemente del sexo.

El análisis global muestra un predominio de estudiantes que aplican una aproximación estratégica, un grupo menor que aplica una aproximación profunda y un porcentaje muy bajo que aplica una aproximación superficial durante su aprendizaje. Estos resultados coinciden con los comunicados por Salas [21] y Escanero et al [22]. No obstante, debe señalarse que para el grupo de Zaragoza [22] el enfoque profundo fue el prioritario, ya que los estudiantes de esa universidad privilegiaron la búsqueda de significado seguido del uso de evidencias, que resultaron ser factores esperados y que teóricamente caracterizan a este tipo de aproximación. Ambas publicaciones señalaron que, a medida que el estudiante subía de nivel, se iba incrementando su aproximación estratégica y, por ende, mejoraba su respuesta a las demandas del contexto, resultados que coinciden con los nuestros. En ese sentido, se señala que ponen en juego otras estrategias relacionadas con la autorregulación, como es el caso del trabajo elaborado en Chile [21], donde también se observó la importancia de los procesos de autosupervisión y regulación de los procesos cognitivos y metacognitivos durante el aprendizaje.

Por otra parte, los resultados mostrados en este trabajo difieren de los comunicados por otros autores que trabajaron específicamente en medicina [17], quienes señalaron que los estudiantes no sólo no se volvían más estratégicos, sino que tendían a ser su- 
Tabla VI. Enfoques de aprendizaje y estrategias de apoyo al procesamiento.

\begin{tabular}{|c|c|c|c|c|}
\hline & & $\begin{array}{l}\text { Aprendizaje } \\
\text { profundo }\end{array}$ & $\begin{array}{l}\text { Aprendizaje } \\
\text { estratégico }\end{array}$ & $\begin{array}{c}\text { Aprendizaje } \\
\text { superficial }\end{array}$ \\
\hline \multirow{2}{*}{ Autoconocimiento } & Correlación de Pearson & $0,541^{a}$ & $0,417^{a}$ & $0,283^{a}$ \\
\hline & Significación (bilateral) & 0,000 & 0,000 & 0,003 \\
\hline \multirow{2}{*}{$\begin{array}{l}\text { Automanejo/ } \\
\text { planificación }\end{array}$} & Correlación de Pearson & $0,571^{a}$ & $0,595^{a}$ & 0,109 \\
\hline & Significación (bilateral) & 0,000 & 0,000 & 0,256 \\
\hline \multirow{2}{*}{$\begin{array}{l}\text { Automanejo/ } \\
\text { regulación } \\
\text { y evaluación }\end{array}$} & Correlación de Pearson & $0,579^{a}$ & $0,513^{a}$ & $0,192^{b}$ \\
\hline & Significación (bilateral) & 0,000 & 0,000 & 0,044 \\
\hline \multirow{2}{*}{ Autoinstrucciones } & Correlación de Pearson & $0,599^{a}$ & $0,532^{a}$ & 0,113 \\
\hline & Significación (bilateral) & 0,000 & 0,000 & 0,241 \\
\hline \multirow{2}{*}{ Autocontrol } & Correlación de Pearson & $0,278^{a}$ & 0,163 & $0,208^{b}$ \\
\hline & Significación (bilateral) & 0,003 & 0,090 & 0,029 \\
\hline \multirow{2}{*}{ Contradistractoras } & Correlación de Pearson & $0,415^{a}$ & $0,369^{a}$ & 0,185 \\
\hline & Significación (bilateral) & 0,000 & 0,000 & 0,053 \\
\hline \multirow{2}{*}{$\begin{array}{l}\text { Interacciones } \\
\text { sociales }\end{array}$} & Correlación de Pearson & $0,497^{a}$ & $0,370^{a}$ & $0,202^{b}$ \\
\hline & Significación (bilateral) & 0,000 & 0,000 & 0,034 \\
\hline \multirow{2}{*}{$\begin{array}{l}\text { Motivación } \\
\text { intrínseca } \\
\text { y extrínseca }\end{array}$} & Correlación de Pearson & $0,370^{a}$ & $0,406^{a}$ & 0,141 \\
\hline & Significación (bilateral) & 0,000 & 0,000 & 0,141 \\
\hline \multirow{2}{*}{$\begin{array}{l}\text { Motivación } \\
\text { de escape }\end{array}$} & Correlación de Pearson & $0,260^{a}$ & $0,207^{b}$ & $0,367^{a}$ \\
\hline & Significación (bilateral) & 0,006 & 0,030 & 0,000 \\
\hline
\end{tabular}

a Correlación significativa al nivel 0,01 (bilateral); ${ }^{b}$ Correlación significativa al nivel 0,05 (bilateral).

perficiales y menos profundos en sus aproximaciones al aprendizaje debido a que, con el paso del tiempo, a diferencia de los resultados comunicados en este trabajo, se interesaban menos por las ideas y hacían menos uso de evidencias durante su aprendizaje. Asimismo, se han observado valores similares en el grupo de estudiantes que aplican enfoque estratégico a lo largo de la carrera.

Newble y Hejka [23], en un estudio sobre las aproximaciones al aprendizaje de estudiantes de medicina, sugirieron que el enfoque profundo, que teóricamente representa ser el más consistente con los objetivos educativos establecidos por cualquier institución educativa, era infrautilizado en favor de los otros enfoques. Las razones para estos autores pa- recen asociarse a la sobredependencia de los procedimientos de evaluación, entre otros, debido a que los estudiantes precisan recordar y reproducir una gran cantidad de información, aspectos que no parecen estar alejados de los resultados de otros estudios.

El grupo de estudiantes de la Facultad de Medicina de la Universidad San Francisco Xavier ha mostrado mayor preferencia por la aplicación de una aproximación estratégica en un $76 \%$ y su relación con el componente 'búsqueda de logros' es alta, pudiendo deberse a tres factores contextuales: el primero, los estudiantes aprobaron las asignaturas en primera instancia, constituyéndose en un grupo con rendimiento académico en busca de éxito; el segundo podría ser el curso, ya que ubicados en el último año de su formación de pregrado responden mejor a la atención de las demandas del contexto, como afirmaron antes otros autores [24]; $y$, finalmente, el tercero sería la nota, ya que siendo el sistema de evaluación sobre 100 puntos y la aprobación mínima de 51, las asignaturas cursadas están regidas bajo el sistema de calificación $\mathrm{B}$ (que asigna una puntuación del $35 \%$ como media de dos pruebas parciales teóricas, un $25 \%$ a la práctica y un $40 \%$ a un examen final), lo que muestra que el sistema asigna una puntuación más alta al componente teórico y finalista del proceso.

Como puede observarse, el contexto y sus características parecen desempeñar un papel importante, así como también la atención a las demandas y características del proceso de enseñanza-aprendizaje, como ya fue analizado por Salim [25] en Tucumán (Argentina), quien señaló que la evaluación y acreditación de saberes determina el enfoque de aprendizaje. Los estudiantes ajustan su enfoque según las demandas (adaptabilidad estratégica). Su intención es comprender (enfoque profundo), pero la evaluación propicia la variación de su enfoque para aprobar (superficial). En esta línea, dentro de los factores contextuales, coincidiendo con Valle et al [26], existe un acuerdo en remarcar el importante papel que desempeñan los criterios de evaluación en la adopción de un determinado enfoque. Para Esquivel et al [19], la forma en que el alumno estudia y aprende se relaciona con las características y cantidad de tareas solicitadas en los cursos, con el contexto educativo y con sus motivos, que a su vez influyen en las estrategias de aprendizaje; sin duda, en la muestra de estudio, las estrategias de codificación como las autopreguntas, aplicaciones y relaciones intracontenido se aplican con mayor preferencia, lo que posteriormente facilitaría el proceso de recuperación de la información solicitada. 
Herrera et al [27] destacaron la importancia de la percepción del aprendizaje, es decir, lo que representa este proceso para el estudiante, y otros autores [22], que analizan la importancia de la percepción social de la evaluación del ambiente educacional preclínico en seis escuelas de medicina en Chile, señalaron que en los últimos años podría orientarse hacia un incremento de la percepción negativa; al parecer, el estudiante incrementa su criticismo o se ve desmotivado con el trascurso del tiempo de su formación. Estos resultados disienten de los encontrados en el grupo de la Universidad San Francisco Xavier, ya que la percepción no es negativa en el último año, sino que se considera más bien una cuestión de oportunidad para los estudiantes que buscan el éxito y los logros.

Con respecto a las correlaciones entre enfoques y estrategias de aprendizaje, el grupo con preferencia por la aproximación estratégica durante el aprendizaje tiene una alta correlación con las estrategias como búsqueda de logros, monitorización de la tarea, organización del estudio y gestión del tiempo, en esa secuencia de predominio en el grupo estudiado; estos resultados variaron en el grupo de quinto año de Zaragoza, donde primaba por una pequeña diferencia el control de la eficacia, seguido de la búsqueda de logros [22]. Al parecer, los estudiantes de la universidad boliviana tienen mayores intereses de logro, aspecto que podría relacionarse con la búsqueda de profesionalización, como también ha citado Salas [21] y, por ende, la posibilidad de inserción laboral y el ascenso social, ya que la gratuidad del sistema universitario boliviano para la formación de los estudiantes se constituye en un factor determinante para este hecho. No se puede dejar de destacar la importancia de las capacidades metacognitivas cuando se habla de monitorización de la tarea, cuyos efectos se observan en las aproximaciones de tipo estratégico con orientación al éxito, que no siempre expresan profundidad. En este caso no se ven muy asociados a las estrategias del enfoque profundo $y$, consecuentemente, su énfasis está puesto también en la atención a las demandas de las tareas y no tanto en un aprendizaje significativo, como han señalado otros autores $[17,22]$.

El grupo de preferencia hacia un aprendizaje profundo aplican las siguientes estrategias en orden de predominio: uso de evidencias, búsqueda de significado y relación e interés por las ideas. Estos resultados son similares a los encontrados en el quinto año de Zaragoza, donde prima por una pequeña diferencia la búsqueda de significado e interés por las ideas. La relación de ideas resulta la estrategia menos aplicada en Zaragoza [22]; sin embargo, en
Chile [17] y en nuestro grupo es una de las estrategias aplicadas que permite al estudiante concatenar sus aprendizajes previos y de relación con otros contenidos durante su aprendizaje.

El grupo reducido de aproximación superficial durante el aprendizaje posiblemente puede deberse al nivel avanzado de su formación de pregrado; en este pequeño grupo, el miedo al fracaso como forma de motivación extrínseca es un resultado también observado en medicina en Zaragoza [22] y, asimismo, por autores como Valle et al [26] y Lara y Corona [28] en otras áreas del conocimiento. Estos resultados muestran que la aproximación al enfoque superficial se relaciona más con la evitación de reforzadores negativos, quizás por la percepción de un contexto amenazante u otras variables personales. Los datos difieren de los obtenidos en Chile [17] en estudiantes de quinto año, por ser un grupo grande donde el factor predominante para ese tipo de aproximación al estudio también es el miedo al fracaso, que es uno de los componentes más frecuentes en este tipo de enfoque, lo que podría sugerir la influencia del sistema educativo universitario en la formación médica, que guarda ciertas características similares en ambos países.

El análisis de la relación entre enfoques y estrategias de aprendizaje en el grupo de estudio parece interesante porque permite estudiar en qué etapa del procesamiento de la información ponen más énfasis los estudiantes ya sea con aproximación estratégica, profunda o superficial.

Con respecto a las estrategias de aprendizaje que se aplican en los tres tipos de enfoques se puede constatar que en estudios anteriores [29] se observaron regularidades que se expresan también en este trabajo. El grupo de estudio del enfoque estratégico aplica preferentemente estrategias de adquisición de la información tales como repaso mental, repaso reiterado y subrayado lineal, estrategias en las que deben apoyar su búsqueda de logros y monitorización de la efectividad. En cuanto a las estrategias de codificación de la información, las aplicadas por este grupo de estudiantes son las autoinstrucciones, la planificación, el automanejo, la regulación y la evaluación, lo que indicaría el uso de estrategias de monitorización efectiva de la tarea que responden al sistema de evaluación que aplica la facultad. Como se señaló anteriormente, los estudiantes con enfoque estratégico ponen todo su esfuerzo en estudiar, controlar el tiempo y el esfuerzo de forma eficiente, encontrando las condiciones y los materiales idóneos para estudiar y monitorizar la efectividad de su estudio, mostrando en el grupo de estudio una preferencia mayor por la búsqueda de logros. Para 
Abalde et al [30], en este tipo de enfoque se observó la planificación buscando eficacia a la hora de la realización de las tareas, acudiendo a toda información posible, hecho que puede relacionarse en este grupo con el automanejo y la regulación.

Como ya se señaló, en este enfoque se observa que la evaluación y acreditación de saberes determina el enfoque de aprendizaje [15]. Los estudiantes ajustan su enfoque según las demandas (adaptabilidad estratégica) [25], aunque no específicamente en esta fase del procesamiento de la información.

Se destaca la relación entre uso de estrategias de recuperación de la información con el enfoque estratégico y también con el profundo, muy relacionado con la búsqueda de logros en el primero, y con la búsqueda de significado y uso de evidencias en el segundo.

En este trabajo, los estudiantes que aplican con preferencia un enfoque profundo apoyan su aprendizaje especialmente con estrategias de codificación, como el uso de autopreguntas, las aplicaciones y las relaciones intracontenido. En cuanto a la importancia del proceso de codificación de la información se destaca el trabajo de Camarero et al [31] en estudiantes de los últimos años, que desvelaron capacidades metacognitivas y de autorregulación durante el aprendizaje, lo que muestra un buen uso de las estrategias de apoyo al procesamiento de la información en el caso del enfoque profundo.

Asimismo, apoyando las conclusiones realizadas por de la Fuente et al [32], no hemos observado relación entre el enfoque superficial y la autorregulación del aprendizaje, tanto con variables de conciencia como de planificación. En ese sentido, la relación con la regulación, como ya se ha comunicado [32], muestra un bajo manejo de los recursos de apoyo necesarios durante la recuperación de la información. El miedo al fracaso es una de las características más destacadas en nuestro grupo y su correlación positiva con el enfoque superficial, como ya ha sido estudiado por otros autores [23], quienes señalaron la importancia de esta estrategia en el caso de aproximaciones superficiales. Asimismo, los estudios antes mencionados [32] han indicado que el enfoque superficial se relaciona positivamente con la regulación externa. En nuestro grupo se destaca también el uso de estrategias de motivación extrínseca, como la motivación de escape.

Otros trabajos $[7,15]$ también han encontrado estos resultados y han mostrado correlaciones similares que hacen afirmar que el enfoque superficial es completamente divergente de los otros dos enfoques. En esa misma línea se ha comunicado [28] además que las estrategias que aplican los estudian- tes con aproximaciones de tipo profundo o estratégico difieren completamente de las aplicadas por el grupo de aproximación superficial, aspecto que también se puede advertir en este estudio.

En ese mismo sentido, se ha aseverado [32] que el enfoque superficial se relaciona positivamente con la regulación externa, mostrando con ello que el enfoque superficial precisa un locus de control externo para responder a la demanda de la tarea; es decir, la conducta del estudiante que privilegia este enfoque en su aprendizaje responde por la exigencia o el temor al otro (profesor, familia u otros) o a las circunstancias que le demandan resultados que no pueden cumplir y por ello se observa un alto miedo al fracaso.

En cuanto a las estrategias de apoyo, como la autorregulación, se aplican como estrategias que facilitan la supervisión del sistema metacognitivo, es decir, la monitorización de la efectividad $[7,17]$ y, en este caso, han sido utilizadas en los tres tipos de enfoques estudiados.

Por otra parte, en cuanto a los estudiantes que aplican un enfoque superficial se podría decir que no aplican las estrategias de subrayado idiosincrático, epigrafiado y repaso mental, y sí aquellas otras como el repaso en voz alta, exploración y repaso reiterado, asociadas más a la memoria a corto plazo, hecho que podría relacionarse con la falta de fomento del análisis, la reflexión, la búsqueda de significado y la relación de ideas, reflejando una memorización sin relacionar contenidos, una característica propia de este enfoque [15]. Se ha referido que este hecho podría asociarse a la búsqueda de aprobación más que a la comprensión de los contenidos [21]. Asimismo, en este enfoque no se han observado correlaciones positivas con las estrategias de planificación de la respuesta; su valor en la recuperación de la información resulta muy importante, sino esencial, en lo referido a este enfoque [33].

El enfoque profundo y el superficial en este estudio son, como ya se señaló en otros estudios [17,34], excluyentes, observándose de forma más evidente durante la fase de codificación de la información. También es de destacar que los enfoques estratégico y profundo aplicados por los estudiantes del internado rotatorio de la Facultad de Medicina tienen una correlación significativa con las estrategias de codificación, mientras que el enfoque superficial no se correlaciona con éstas.

En conclusión, la aproximación al aprendizaje predominante en el último año de la formación del médico en la universidad analizada es la estratégica, y las estrategias con las que se relaciona más este tipo de enfoque son las de recuperación de la 
información; asimismo se puede observar la relación del aprendizaje estratégico y las aspiraciones de éxito y logro del estudiante de últimos niveles. Si bien no existen diferencias significativas en las estrategias que aplican las aproximaciones estratégica y profunda, es el componente de logros una de las características que se destaca en el grupo de estudiantes con preferencia estratégica, mostrando la importancia del componente motivacional en la conducta del aprendiz. Se debe insistir, a partir de estas primeras aproximaciones, en la necesidad de que futuros estudios profundicen sobre la influencia de otras variables contextuales como sistema educativo, evaluación y autorregulación, ya que podrían orientar los procesos de mejora de la enseñanzaaprendizaje que se desarrollan en las aulas universitarias. No obstante, para facilitar el aprendizaje de los estudiantes de este centro se deben llevar a la práctica las conclusiones obtenidas a partir de los resultados comunicados en este trabajo.

\section{Bibliografía}

1. Escanero JF, Guerra M, Soria MS. Elementos para el diseño de una práctica de metacognición: conocimientos del cómo. Universidad de Zaragoza, Serie LEFIS 2011; 13: 225-46.

2. Roosta M, Suárez L, Rodríguez J. Bolivia: diagnóstico nacional sobre las políticas de investigación en las universidades. Instituto Internacional de Educación Superior para América Latina y el Caribe (IESALC-UNESCO)/Universidad Tecnológica Privada de Santa Cruz (UTEPSA); 2005.

3. Universidad Mayor Real Pontificia de San Francisco Xavier de Chuquisaca. Modelo Académico. Sucre, Bolivia; 2011.

4. Daza R, Roca V. Estudio de la educación superior en Bolivia. Proyecto informe de la educación superior en Iberoamérica. Santiago de Chile: CINDA; 2006. URL: http://www.cinda.cl/ download/informes_nacionales/bolivia.pdf.2006.

5. Santa Cruz J. La educación superior en el marco de la descentralización: contexto y perspectivas. Ministerio de Hacienda. Red de Análisis Fiscal. URL: http://www.forodac. org.bo/upload/301_Ed_Superior.pdf.

6. CEUB. XI Congreso Nacional de Universidades. Lineamientos para el desarrollo curricular. Bolivia; 2010.

7. Congreso Nacional de Educación, Documento de trabajo I. Resumen de los Congresos Departamentales. La Paz: Industrias Lara Bisch; 2005.

8. Torre JC. Una triple alianza para un aprendizaje universitario de calidad. Madrid: Universidad Pontificia Comillas; 2007.

9. Pozo J, Monereo C, eds. El aprendizaje estratégico. Madrid: Santillana; 1999

10. Poggioli L. Estrategias cognoscitivas: una revisión teórica y empírica. In Poggioli L, Puente A, Navarro A, eds. Psicología cognitiva. México: McGraw-Hill; 1995.

11. OCDE. Informe PISA 2009. Aprendiendo a aprender. Implicación, estrategias y prácticas de los estudiantes. Vol. III. Madrid: Santillana Educación; 2010.

12. Bernard JA. Análisis de estrategias de aprendizaje en la universidad. Investigación. Zaragoza: Instituto de Ciencias de la Educación, Universidad de Zaragoza; 1992.

13. Monereo C. Estrategias de enseñanza y aprendizaje. Formación del profesorado y aplicaciones en la escuela. Barcelona: Graó; 1994.

14. Román JM. Procedimiento de aprendizaje autorregulado para universitarios. La estrategia de lectura significativa de textos. Valladolid: Facultad de Educación, Universidad de Valladolid. URL: http://repositorio.ual.es/jspui/bitstream/ 10835/675/2/Art_3_33_spa.pdf.

15. Entwistle N. Questionnaire on approaches to learning and studying. Edinburgh: Centre for Research on Learning and Instruction, University of Edinburgh; 1993.

16. Román JM, Gallego S. ACRA, escala de estrategias de aprendizaje. Madrid: TEA Ediciones; 2001.

17. Díaz G, Mora S, Escanero JF. Estilos, enfoques y contexto de aprendizaje. Escuela de Medicina de la Universidad de Chile. Zaragoza: Prensas Universitarias de Zaragoza; 2011.

18. Biggs J, Kember D, Leung DP. The revised two factor Study Process Questionnaire: R-SPQ-2. Br J Educ Psychol 2001; 71: 133-49.

19. Esquivel J, Rodríguez MC, Padilla VM. Enfoques hacia el aprendizaje, motivos y estrategias de estudiantes de las carreras de enfermería, ingeniería y organización deportiva. Rev Pediatr Caracas 2009; 30: 309-31.

20. Lafuente JV, Escanero J, Manso JM, Mora S, Miranda T, Castillo $\mathrm{M}$, et al. El diseño curricular por competencias en educación médica: impacto en la formación profesional. Educ Med 2007; 10: 86-92.

21. Salas R. Enfoques de aprendizaje entre estudiantes universitarios. Estud Pedagog 1998; 24: 59-78.

22. Escanero JF, Mora S, Arce J, Bianchi AR, Díaz G, Gargiulo PA, et al. Estilos de aprendizaje y currículum: propuesta de mejora. Facultad de Medicina de Zaragoza. Zaragoza: Prensas Universitarias de Zaragoza; 2009.

23. Newble DI, Hejka EJ. Approaches to learning of medical students and practicing physicians: some empirical evidence and its implications for medical education. Educ Psychol 1991; 11: 333-42.

24. Cabanach RG, Valle A, Piñeiro I, Rodríguez S, Núñez IC. El ajuste de los estudiantes con múltiples metas a variables significativas del contexto académico. Psicothema 1999; 11: 313-23.

25. Salim R. Motivaciones, enfoques y estrategias de aprendizaje en estudiantes de bioquímica de una universidad pública argentina. REDIE, Revista Electrónica de Investigación Educativa 2006; 8. URL: http://redie.uabc.mx/vol8no1/ contenido-salim.html.

26. Valle A, González R, Núñez JC, Suárez JM, Piñeiro I, Rodríguez S. Enfoques de aprendizaje en estudiantes universitarios. Psicothema 2000; 12: 368-75.

27. Herrera C, Pacheco J, Rosso F, Cisterna C, Aichele D, Becker S, et al. Evaluación del ambiente educacional preclínico en seis escuelas de medicina en Chile. Rev Med Chile 2010; 138: 677-84.

28. Lara J, Corona E. Estrategias de aprendizaje y rendimiento académico: el caso de la Facultad de Ingeniería de la UAS. Centro de Investigaciones y Servicios Educativos. Universidad Autónoma de Sinaloa. México: Universidad Autónoma de Sinaloa; 2005.

29. Paredes P. Una propuesta de incorporación de los estilos de aprendizaje a los modelos de usuario en sistemas de enseñanza adaptativos [tesis doctoral]. Madrid: Universidad Autónoma de Madrid; 2008. URL: http://arantxa.ii.uam.es/ pparedes/ tesis.pdf.

30. Abalde E, Barca A, Muñoz JM, Fernando M. Rendimiento académico y enfoques de aprendizaje: una aproximación a la realidad de la enseñanza superior brasileña en la Región Norte. Rev Invest Educ 2009; 27: 303-19.

31. Camarero FJ, Martín del Buey F, Herrero J. Estilos y estrategias de aprendizaje en estudiantes universitarios. Psicothema 2000; 12: 615-22.

32. De la Fuente J, Pichardo MC, Justicia F, Berbén A. Enfoques de aprendizaje, autorregulación y rendimiento en tres universidades europeas. Psicothema 2008; 8: 705-11.

33. Pérez MV, Valenzuela MF, Díaz A, González JA, Núñez JC. Disposición y enfoques de aprendizaje en estudiantes universitarios de primer año. Univ Psychol Bogotá 2011; 10: 441-9.

34. González R, Valle A, Suárez JM, Fernández A. Un modelo integrador explicativo de las relaciones entre metas académicas, estrategias de aprendizaje y rendimiento académico. Rev Invest Educ 1999; 17: 47-70. 